\title{
Assessing the impact of an online training program on research performance of students and faculty members
}

\author{
Dr. H. T. Jadhav ${ }^{1}$, Mr. P. D. Bamane ${ }^{2}$ \\ ${ }^{1}$ Electrical Engineering Department, Rajarambapu Institute of Technology, Islampur, Sangli, Maharashtra \\ ${ }^{2}$ Electrical Engineering Department, Rajarambapu Institute of Technology, Islampur, Sangli, Maharashtra \\ ${ }^{1}$ htj@ritinida.edu \\ ${ }^{2}$ pradip.bamane@ritindia.edu
}

\begin{abstract}
The short term training programs, in engineering institutes, are planned to provide a platform to the students, research scholars and faculty members to help them identify and formulate research problems in latest technical areas. The training programs, in face-toface or online mode, designed for specific fields of research need to be evaluated to assess whether or not such programs serve the intended purpose. This study examined the effectiveness of an online training program in terms of response of participants to engage in research activities in the field of application of evolutionary and swarm intelligence techniques to solve engineering optimization problems. A total of 643 students $(37.3 \%)$ and faculty members $(62.7 \%)$ from different technical institutes/universities in India with different engineering domains completed two one week training programs through online mode in the month of June 2020 and 301 $(46.81 \%)$ were followed up through online mode to assess impact of these programs on improvement in the research work of participants. The results of the study indicate that more than $65.2 \%$ participants out of 290 successfully formulated and implemented at least one method of engineering optimization to solve the research problem in their area of interest within a period of just one month. Moreover, the study shows that $16.4 \%$ participants have their research paper under review process while $18.4 \%$ participants have requested for another training program to improve their knowledge for better understanding of topics in the field of engineering optimization. The other findings suggest that the online training programs for students and faculty members were useful and the impact on students and research scholars was significant in improving knowledge and attitude to learn other methods of engineering optimization available in literature. The overall experience is that such online training programs can be more effective if designed with specific research problems and by encouraging participants to collaborate with other participants.
\end{abstract}

\section{Keywords: Training Program, Workshop, Evaluation}

\section{Introduction}

The research publications in scientific and scholarly journals is an important factor which reflects the involvement of students, research scholars and faculty members in research activities. The research culture in universities and institutions in developed countries is very well established. However, in the developing countries the teaching learning process is better organized but the research culture is not yet well developed barring few top ranked institutes. Most universities and institutes facilitate training programs for their employees to upgrade their domain knowledge or research aptitude. However, assessment of effectiveness of such training programs is not carried out to study the impact of such trainings. Online training programs have become popular over the past twenty years due to development in the field of internet technology and fastest growing sector in the fieldof higher education and research.

The effectiveness oftraining programsdepends onthe use of diverse teaching and learning methods (Sarikaya, O 2010). In general, training programs are evaluated by using questionnaire or grading performance by the observer (Hewson, M. G 2001). In some of the researchinvestigations, the impact of training programs is evaluated by conducting interviews of participants on one to one basis.(Musal, B 2002). The researchers are always in hunt or need the techniques to find the solutions on the defined problem. One of the solutions for this is the short or long term training or the workshops. So, undergraduate and postgraduate students, research scholars and faculty members from different universities are always in search of the short term or long term training programs. All may have very good content knowledge but they always require skills to do research in their respective area. The short or long term training program fulfils that need.

A workshop or training program is a step which help participants who are having research experience or not, and also who is already doing research or formulating research problem. The research shows that the training program provides professional competencies in faculties (Eckstrom et al. 2006). The research also shows that short or long term training programs or workshops are most used medium for professional development. But the effectiveness of such training programs or workshops is always a concern (Berbano et al. 2006). Many studies also stated that these training programs or workshops will be effective if some of the existing techniques can be used (Baral et al. 2007) (Houston at al. 2004) (Leslie et al. 2013). It is really difficult to measure the outcome of the training program or workshops. Most frequent measuring criteria to check the participant satisfaction is feedback. Some of the studies developed tools to measure the effectiveness of these workshops or trainings. But most 
important factor of these tools is resistance from the participants to participate in the activity (Eckstrom et al. 2006).

Many efforts are taken to investigate effectiveness of the workshop or training programs research but still this is not sufficient. So evaluation is an essential process to check the effectiveness and to assess whether desired objective of the program is achieved or not after completion of the workshop or training program. (Musal et al. 2008) (Morrison 2003) (Durning et al. 2007).

For the evaluation of training program or workshop many methods have been proposed. Donald Kirkpatrick model is one pioneer and basic model which serves the purpose of evaluation (Kirkpatrick \& Kirkpatrick 2006). This model has been in use from last thirty years (Bates 2004).

This model consists of four important levels which include reaction, learning, behaviour and results. This model is represented in the Fig. 1. Each level in this model has an impact on the next level. The first level represents the feedback of participants who participated in the workshop or training program. Through this level the organizer get idea about participant satisfaction level (Kirkpatrick \& Kirkpatrick 2005). Second level is called as learning level. Through this level organizer can get idea about to what extent the participant got knowledge, skill and attitude from the training program or workshop organized (Knowles et al. 1998; Ehlers \&Schneckenberg 2010). Third level is called as behaviour. This level measures to what extent the behaviour of participantshas changed after attending the workshop or training program. Fourth level is called as results. This is very important level. This level is about outcome of the project (Kirkpatrick \& Kirkpatrick 2007). It has also found that the evolution model should be developed according to the need (Fitzpatrick et al. 2004). It should give emphasis to both the process and result (Musal et al. 2008).

The evaluation method can be considered as ideal if it is reliable, valid and inexpensive. This study also aims to examine the effectiveness of an online training program in terms of response of participants to engage in research activities in the field of application of evolutionary and swarm intelligence techniques to solve engineering optimization problems.

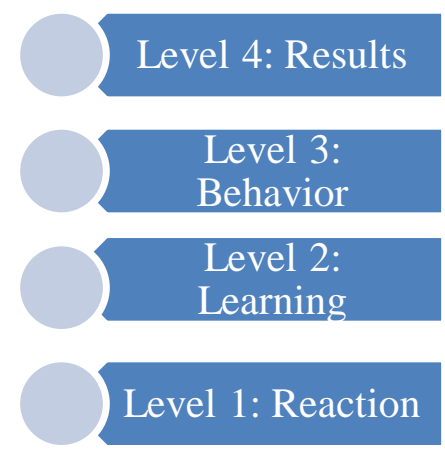

Fig 1: Donald Kirkpatrick model

\section{Methods}

A. The workshop details:

Two workshops were conducted in the period June July, 2020. First training program titled, "4th One Week Short Term Training program on, Implementation of Advanced Optimization Techniques using MATLAB" conducted from $1^{\text {st }}$ June to $6^{\text {th }}$ June, 2020 and second training program titled, "One-Week Online Training Program on, Implementation of Multi-objective Optimization Algorithm(NSGA -II) using MATLAB" conducted from $29^{\text {th }}$ June to $3^{\text {rd }}$ July, 2020. The objective of the first short term training program were participants will be able to implement suitable evolutionary optimization technique to solve problem identified and participant will able to write MATLAB code of Meta heuristic technique.

The objectives of the second short term training program were to help participants understand concept of Genetic algorithm \& NSGA -II, to enable participants implement GA \& NSGA - II using MATLAB programming language and to demonstrate the philosophy of GA \& NSGA-II through tutorial.

These two short term training programs were designed to help researchers to understand different optimization methods and their applications in engineering. All sessions in both the short term training program were supported by tutorial and lab for programming work. These two short term training programs were open for undergraduate, post graduate, research scholar faculty members and industry personal. The objectives of this research work is to assess the impact of these workshop on the improvement of research aptitude among participants

\section{B. Evaluation tools}

The four levels Kirkpatrick model is used to evaluate the training program. It is evaluated on the front of formative and summative evaluation. The formative evaluation used to find effectiveness of the program. It includes identifying the strength and weakness, organization of training program, content quality, delivery and interaction between resource person and participants. The summative evaluation used to evaluate the impact of training program on the participant. It includes evaluation of whether participant get the knowledge, skills and also whether improved his behaviour to produce the research publication or not.

\section{Data collection}

For evaluation purpose data is collected. To collect data the quantitative and qualitative methods are used. For both the workshop four evolution tools were used. It includes entry test (test prior to start of short term training), feedback during each session, overall feedback of workshop, exit test (test after completion of short term training) and feedback after one month (after completion of training) to check the behavioural change. These five tools are used for formative and summative evaluation. The tools such as entry test, exit test and feedback after 
one month are used for summative evaluation. The tools such as feedback during each session and overall feedback of workshop are used for formative evaluation. The entry test and exit test are developed based on the objectives of the respective short term training program.

\section{Result and discussion}

A total of 643 students (37.3\%) and faculty members $(62.7 \%)$ from different technical institutes/universities in India with different engineering domains completed two one week training programs through online mode in the month of June 2020 and 301 (46.81\%) were followed up through online mode to assess impact of these programs on improvement in the research work of participants.

Table 1: Tools used for evalution of training program

\begin{tabular}{|c|l|l|}
\hline $\begin{array}{c}\text { Sr. } \\
\text { no. }\end{array}$ & \multicolumn{1}{|c|}{ Tool used } & \multicolumn{1}{|c|}{ Scale/score } \\
\hline 1 & Entry Quiz & $\begin{array}{l}\text { Average score: 28.12 out of } \\
50\end{array}$ \\
\hline 2 & $\begin{array}{l}\text { Overall Feedback } \\
\text { about the training } \\
\text { program }\end{array}$ & Average score: 4.37 out of 5 \\
\hline 3 & Exit Quiz & $\begin{array}{l}\text { Average score: 37.69 out of } \\
50\end{array}$ \\
\hline 4 & $\begin{array}{l}\text { Feedback after one } \\
\text { month }\end{array}$ & $\begin{array}{l}\text { The questionnaire and its } \\
\text { respective responses are } \\
\text { mentioned in the Fig. 3to } \\
\text { Fig. 17. }\end{array}$ \\
\hline
\end{tabular}

The domains of these 301 responses are shown in Fig.3.These training programs were related to engineering problems so all the participant were from engineering domain only. Since the aim of this training program was to inculcate the research aptitude in the participants. Therefore prior to the training program the optimization related papers were distributed with the participants. The purpose behind giving these papers was participants can see the formulation and solve the problem mentioned in the paper by using the techniques discussed in the training. This is also seen through the satisfaction score obtained in training programs for overall feedback. The satisfaction score is given in the Fig. 2. The average satisfaction score obtained is 4.37. The satisfaction score range is form 1 to 5 , where 1 represents worst and 5 represents excellent.

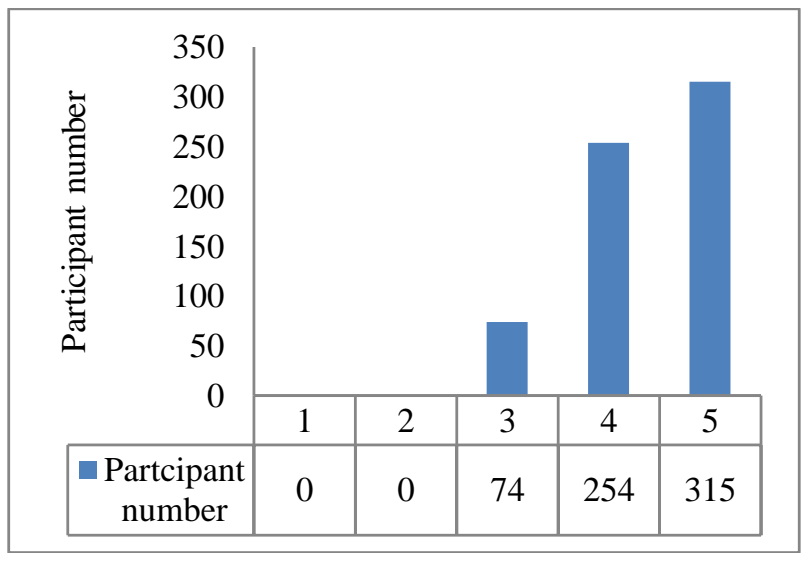

Fig 2: Satisfaction score

To check whether behaviour of participant is changed or not feedback after one month was taken. The Fig. 3 to Fig. 15 gives idea about the behavioural change. There were two workshop organized and out of the 301 students 170 attended first training program and 98 attended second one, 55 attended both. This information is given Fig. 3 .

Fig. 4 shows that the current status of participants. Out of 301, 71 were undergraduate students, 33 post graduate students, 71 research scholars, 146 faculty members, 1 MBBS doctor and 1 Electrical Engineer trainee.

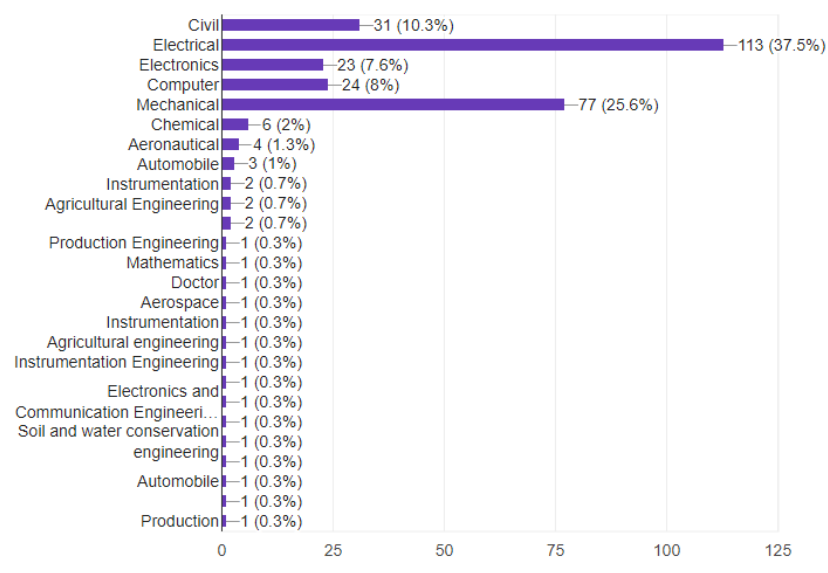

Fig3: Participant domain

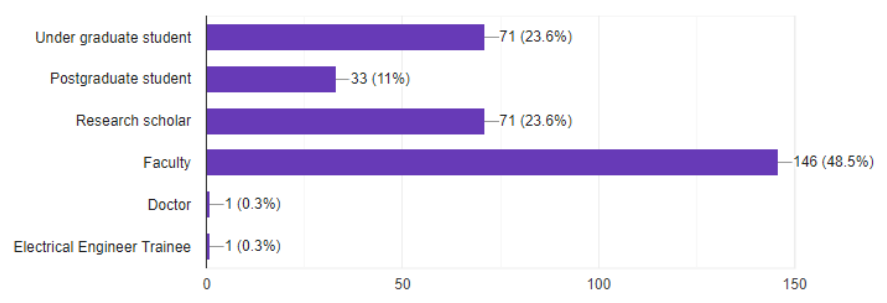

Fig 4: Participant status 


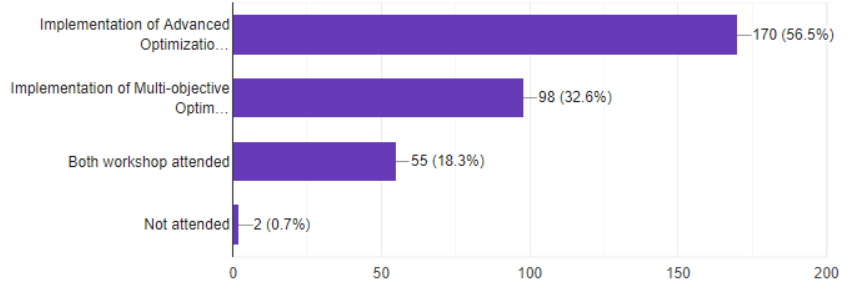

Fig 5: Workshop to which you enrolled (June/July 2020).

The training programs organized were related to the optimization technique. The outcome of the program can be measurable only if participant able to find research problem in the field of optimizationand also able to formulate the optimization problem. So to check whether the participant really identified the problem and formulated the same,questionnaire was added in the feedback form. The result shows that $81.7 \%$ participants could identify the research problem and $39.2 \%$ could able formulate the same also. The results are shown in Fig. 6 and Fig. 7 respectively.

To check whether the behaviour of participant is changed or not, aquestionnaire was also added such as "After attending the workshop, have you started working on the research problem, you identified?". The result shows that around $65.4 \%$ started their research work. The result is shown in Fig. 8

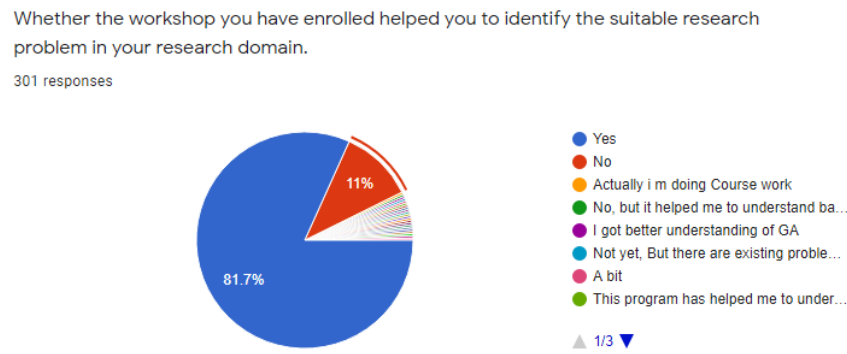

Fig 6: Whether the workshop you have enrolled helped you to identify the suitable research problem in your research domain

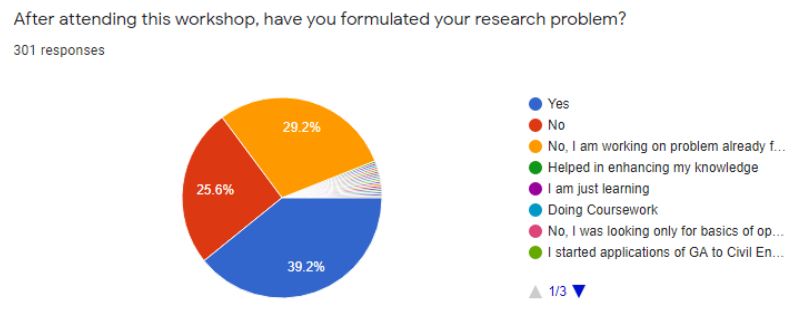

Fig 7: After attending this workshop, have you formulated your research problem?

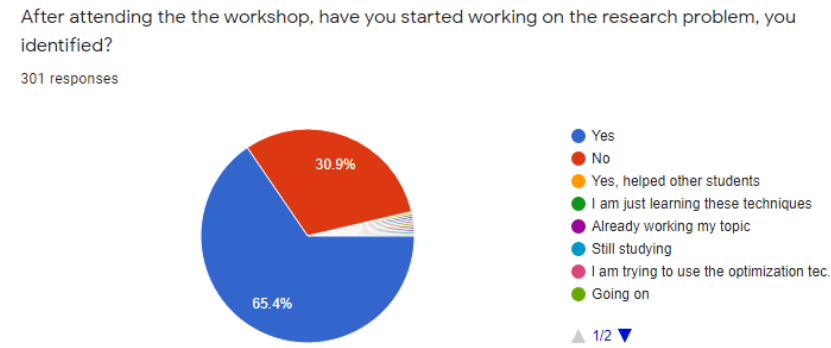

Fig 8: After attending the workshop, have you started working on the research problem, you identified?

The questionnaire was added in the feedback form to check whether participant submitted the research work for publication. The result shows that around $11.6 \%$ could able to do that. The result is shown in the Fig. 9.

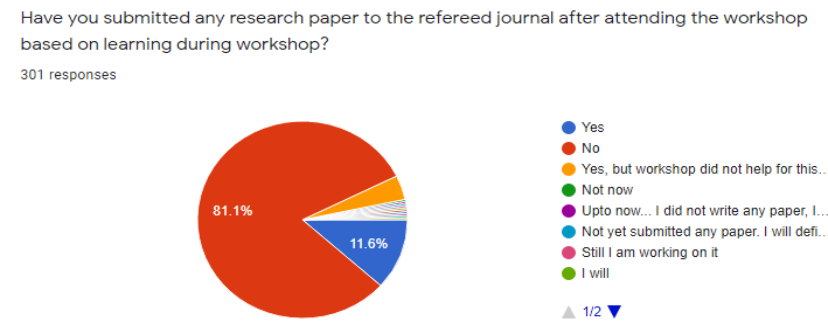

Fig 9: Have you submitted any research paper to the refereed journal after attending the workshop based on learning during workshop?

To check whether the paper submitted is under revision or rejected or under review process questionnaire was added. The result shows that $16.6 \%$ under revision and $16.6 \%$ were under review process. The result is shown in the Fig. 10.

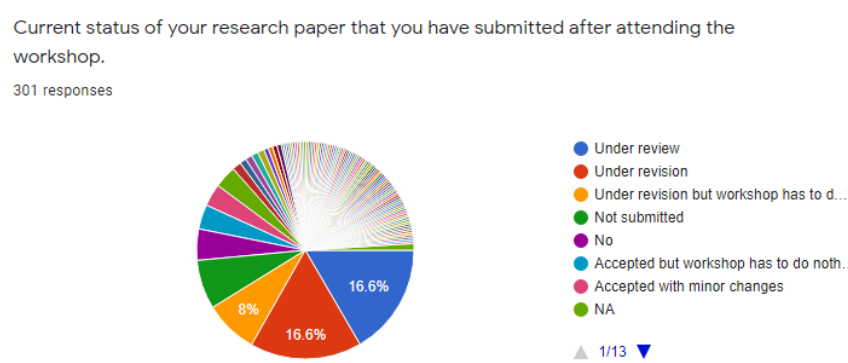

Fig 10: Current status of your research paper that you have submitted after attending the workshop. 
To check whether this training program made any improvement in the research work of the participant,questionnaire was also added. The result shows that $63.5 \%$ participants feel training program improved the quality of their research work after attending the program and this is shown in Fig. 11.

If you are already working in the field of optimization, due you think this workshop helped you to improve the quality of research in your research domain?

301 responses
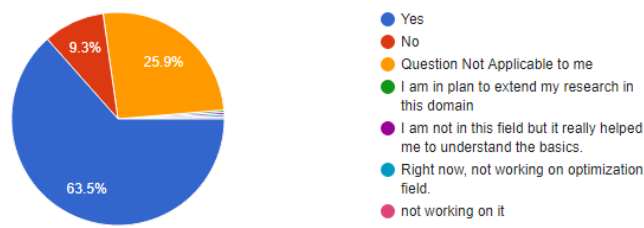

Fig 5: If you are already working in the field of optimization, due you think this workshop helped you to improve the quality of research in your research domain?

Some specific questionnaire was added to check the behavioural change in the undergraduate, research scholar and faculty members. The result shows that $32.6 \%$ undergraduates feel research aptitude is embedded after attending the programs and it is shown in Fig. 12. To check whether research scholar formulated and solve the optimization problem after attending the training programs questionnaire was added. The result shows that $46.8 \%$ research scholar feel they could able formulate and solve the research problem after attending the training programs. This result is shown in Fig. 13

If you are under graduate student, then do you feel research aptitude is now embedded in you after attending this workshop

301 responses
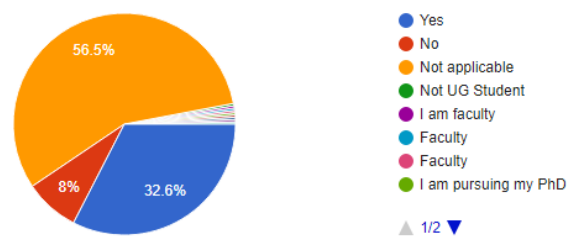

Fig 6: If you are under graduate student, then do you feel research aptitude is now embedded in you after attending this workshop

If you are research scholar, then do you feel this workshop helped you to find the researc problem and able to solve it after attending this workshop.

301 responses
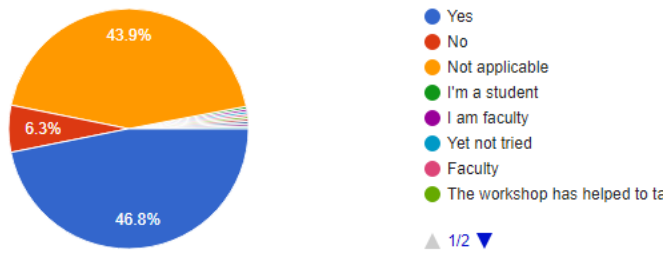

$1 / 2 \mathbf{\nabla}$

Fig 7: If you are research scholar, then do you feel this workshop helped you to find the research problem and able to solve it after attending this workshop.
Also to check whether the training program made any difference between faculty members, the questionnaire was also added. They are basically two cases. One the faculty member doing research in the field of optimization and second one is faculty who is not doing research in the field of optimization. So to check this different question were added in the feedback form. The Fig. 14 and Fig. 15 show the result for these two cases. According to result it is found that $55.8 \%$ faculty members who are doing research work in the same field feel the training program give insight in the optimization field. This result is shown in Fig. 15. 45.2\% faculty members whose research domain are different but after attending the training program feels they got insight in the optimization domain. This result is shown in Fig. 15.

If you are faculty and doing the research in the filed of optimization then do you really feel this workshops had given more insight in to the field of optimization to do future research work.

301 responses
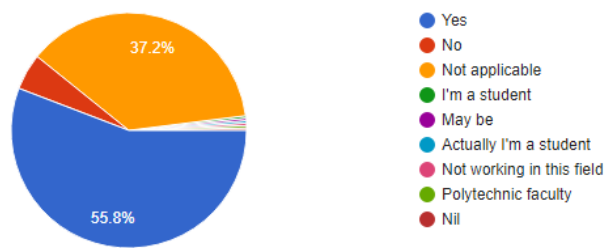

Fig 8: If you are faculty and doing the research in the field of optimization then do you really feel this workshops had given more insight in to the field of optimization to do future research work.

If you are faculty and not doing research work in the filed of optimization then do you really feel this workshops had given enough insight in to the field of optimization to do research work in the field of optimization.

301 responses

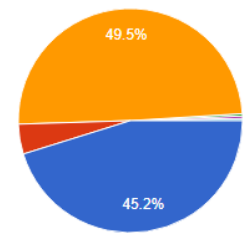

- Yes

- Not applicable

- I'm a student

Doing work on optimization

Now I'm student

$45.2 \%$

Fig 9: If you are faculty and not doing research work in the field of optimization then do you really feel this workshops had given enough insight in to the field of optimization to do research work in the field of optimization.

One more important purpose of this workshop was to utilize the lockdown period effectively. In view of this training program was developed and because of that the objective of the training program was to cultivate research aptitude.

One more advantage of the optimization domain is that they can publish work with or without experiment base. So to check whether these two training program serves the purpose or not questionnaire was also added. The result shows that $77.1 \%$ participants feel that this training program was good idea to utilize lock down period for research work based on optimization method since it doesn't require laboratory facility. This result is shown in Fig. 16. 
Do you think that such workshops can be good idea to utilize lock down period for research work based on optimization methods since it does not require Laboratory facilities and yet we can doe research work for publication in journals? 301 responses
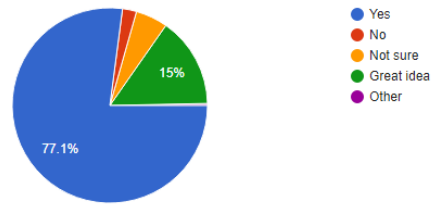

Fig 10: Do you think that such workshops can be good idea to utilize lock down period for research work based on optimization methods since it does not require Laboratory facilities and yet we can do research work for publication in journals?

Recommendation of the training program to others is also reflects the satisfaction level of the participants. So to check that questionnaire was added. The result shows that $48.5 \%$ participant feel that the recorded videos of the training program can be share with their friends or colleagues. This result is shown in the Fig. 17.

Did you share the recorded video links with others to help them learn more about MATLA implementation of Optimization Methods?

301 responses
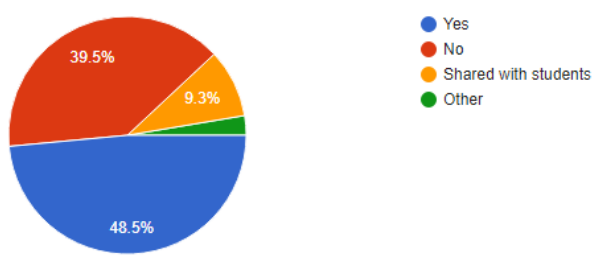

Fig 11: Did you share the recorded video links with others to help them learn more about MATLAB implementation of Optimization Methods?

\section{Conclusions}

The short term training programs are planned to provide a platform to the students, research scholars and faculty members to help them identify and formulate research problems in latest technical areas. But main concern of this training program is to evaluate and to assess whether or not such programs serve the intended purpose.

This paper presents and studies the effectiveness of an online training program in terms of response of participants to engage in research activities in the field of application of evolutionary and swarm intelligence techniques to solve engineering optimization problems.

The results of the study indicate, $65.4 \%$ participants able to formulate and implement engineering optimization problem in the period of one month after completion of training program. The result also indicates that $11.6 \%$ submitted their research work and out of that $16.6 \%$ researcher's paper are under review now.

The result also shows that the training program helped students, research scholars and faculty members to do research work in the field of optimization. The overall experience is that such online training programs can be more effective if designed with specific research objective and with proper plan.

The result presented in this research paper are based the data collected after one month of the completion of the training program. However the study shows that $32.6 \%$ student participants feels that the research aptitude is embedded after attending the training program. Moreover, the $46.8 \%$ research scholar could successfully able to formulate the research problem in the field of optimization after attending the training program. Hence it is likely that these participants will successfully complete the research work in future.

\section{Acknowledgement}

This two training programs were organized by Electrical Engineering Department of Rajarambapu Institute of Technology, Islampur. We could organize this training program because of the constant support from Dr. Mrs. S. S. Kulkarni, Director, Dr. S. K. Patil, Dean Academics, Dr. V. N. Kalkhmabkar, HoD Electrical and all teaching and nonteaching staff members of Electrical Department.

\section{Reference:}

Sarikaya, O., Kalaca, S., Yeğen, B. Ç.,\& Cali, S. (2010). The impact of a faculty development program: evaluation based on the self-assessment of medical educators from preclinical and clinical disciplines. Advances in physiology education, 34(2), 35-40..

Hewson, M. G., Copeland, H. L., \&Fishleder, A. J. (2001). What's the use of faculty development? Program evaluation using retrospective self-assessments and independent performance ratings. Teaching and Learning in Medicine, 13(3), 153-160.

Musal, B., Abacioglu, H., Dicle, O., Akalin, E., Sarioglu, S., \&Esen, A. (2002). Faculty Development Program in DokuzEylül School of Medicine: In the process of curriculum change from traditional to PBL. Medical Education Online, 7(1), 4533.

Eckstrom E, Homer L, Bowen JL. Measuring outcomes of a one-minute preceptor faculty development workshop. Journal of general internal medicine. 2006;21(5):410-4.

Berbano EP, Browning R, Pangaro L, Jackson JL. The impact of the Stanford Faculty Development Program on ambulatory teaching behavior. Journal of general internal medicine. 2006;21(5):430-4.

Baral N, Paudel BH, Das BK, Aryal M, Das BP, Jha N, et al. An evaluation of training of teachers in medical education in four medical schools of Nepal. Nepal Med Coll J. 2007;9(3):157-61.

Houston TK, Clark JM, Levine RB, Ferenchick GS, Bowen JL, Branch WT, et al. Outcomes of a national 
faculty development program in teaching skills: prospective follow-up of 110 medicine faculty development teams. Journal of general internal medicine. 2004;19(12): 1220-7.

Leslie K, Baker L, Egan-Lee E, Esdaile M, Reeves S. Advancing faculty development in medical education: a systematic review. Academic medicine: journal of the Association of American Medical Colleges. 2013;88(7):1038-45.

Musal B, Taskiran C, Gursel Y, Ozan S, Timbil S, Velipasaoglu S. 2008. An example of program evaluation project in undergraduate medical education. Edu Health 21(1):1-7.

Morrison J. 2003. ABC of learning and teaching in medicine: Evaluation. Br Med J 326: 385-387.

Durning SJ, Hemmer P, Pangaro LN. 2007. The structure of program evaluation: An approach for evaluating a course, clerkship or components of a residency or fellowship training program. Teach Learn Med 19(3):308318.

Kirkpatrick DL, Kirkpatrick JD. 2006. Evaluating training programs: The four levels. 3rd ed. San Francisco: BerrettKoehler Publication.

Bates R. 2004. A critical analysis of evaluation practice: The Kirkpatrick model, and the principle of beneficence. Eval Program Plann 27:341-347.

Kirkpatrick DL, Kirkpatrick JD. 2006. Evaluating training programs: The four levels. 3rd ed. San Francisco: BerrettKoehler Publication.

Knowles MS, Holton EF, Swanson RA. 1998. The adult learner. 5th ed. Houston: Gulf Publishing Company.

Ehlers UD, Schneckenberg D. 2010. Changing cultures in higher education: Moving ahead to future learning. Heidelberg, New York: Springer.

Kirkpatrick DL, Kirkpatrick JD. 2007. Implementing the four levels. San Francisco: Berrett-Koehler Publication.

Fitzpatrick JL, Sanders JR, Worthen BR. 2004. Program evaluation: Alternative approaches and practical guidelines. 3rd ed. Boston: Allyn and Bacon 